Please do not remove this page

RMIT

UNIVERSITY

\title{
Space-time OFDM with adaptive beamforming: Performance in spatially correlated channels
}

Lin, Kevin; Hussain, Zahir; Harris, Richard

https://researchrepository.rmit.edu.au/esploro/outputs/9921858064101341/filesAndLinks?institution=61RMIT_INST\&index=null

Lin, K., Hussain, Z., \& Harris, R. (2004). Space-time OFDM with adaptive beamforming: Performance in spatially correlated channels. IEEE TENCON 2004, 617-620.

https://doi.org/10.1109/TENCON.2004.1414671

Published Version: https://doi.org/10.1109/TENCON.2004.1414671

Repository homepage: https://researchrepository.rmit.edu.au

(c) 2004 IEEE. Personal use of this material is permitted. However, permission to reprint/republish this material for advertising or promotional purposes or for creating new collective works for resale or redistribution to servers or lists, or to reuse any copyrighted component of this work in other works must be obtained from the IEEE.

Downloaded On 2023/04/26 21:36:18 +1000 


\title{
SPACE-TIME OFDM WITH ADAPTIVE BEAMFORMING: PERFORMANCE IN SPATIALLY CORRELATED CHANNELS
}

\author{
Kevin H. Lin, Zahir M. Hussain, and Richard J. Harris \\ Centre for Advanced Technology in Telecommunications (CATT) \\ School of Electrical and Computer Engineering \\ RMIT University, Melbourne, Victoria 3000, Australia \\ Emails: s9510490@student.rmit.edu.au,zmhussain@ieee.org, richard@catt.rmit.edu.au
}

\begin{abstract}
Space-time block coding (STBC) has proved to be an effective means of exploring diversity branches and improving system performance. In a previous work we proposed an extension to that where the STBC was combined with adaptive eigenbeamforming, an approach that takes an advantage of spatial channel correlation and can provide further improvement for the overall system performance. In this paper, we investigate this new transmission structure for broadband orthogonal frequency division multiplexing (OFDM) systems in frequency-selective channels. Numerical analysis showed that systems employing this scheme would not undergo any diversity losses as it maintains the maximum achievable diversity advantage of spacetime (ST) codes in realistic channel conditions. Simulation results showed that the proposed structure has a significant improvement in bit/symbol error rate performance in a spatially correlated channel over systems that utilize only space-time coding.
\end{abstract}

\section{INTRODUCTION}

Over the last few years, transmit diversity techniques had become one of the popular research areas in wireless communications. The work in [1] is a well-known STBC scheme that demonstrated the capability of creating additional spatial and temporal diversity branches and hence provide considerable gain in error rate performance in a multiple input multiple output (MIMO) channel configuration. Then later it was generalized and extended in [2] as orthogonal-STBC (OSTBC). In the context of transmit diversity, adaptive beamforming is a different approach to enhance the performance of wireless systems employing multiple transmit antennas. Systems with a structure that combines these two transmission techniques have shown in [3] that it can provide great improvement on error rate performance in a spatially correlated channel environment. In this paper, the idea in [3] is further extended and applied for broadband OFDM systems.

The principal idea in this new transmission scheme is the transmission of data symbols that is first spacetime (ST) encoded then transmitted in the direction of

0-7803-8560-8/04/\$20.00(c)2004IEEE strongest signal path seen by the transmitter. Based on uplink path angles estimates, eigen-analysis on channel spatial covariance matrix can be performed at the transmitter and hence achieve the decorrelation of spatially correlated signal paths. Therefore, the resultant effect of this transmission technique is the maximization of signal-to-noise ratio (SNR) (between the transmitted signal and channel noise) at the receiver.

Other benefits of using this processing structure is the elimination of the need for feedback (uplink) signalling of the channel estimates from the receiver to the transmitter. Hence, it reduces the computation and the complexity of overall transmission system that is usually required in other adaptive beamforming schemes. It becomes more attractive especially for a transmitter with a large number of antennas. It should be noted that our proposed transmission scheme is different from other similar arrangements in the area of combining beamforming with diversity coding. The difference lies in the method and the location of computing transmit antenna weights.

Notation used in this paper: $(.)^{*},(.)^{T}$, and $(.)^{H}$ are complex number conjugate, transposition, and Hermitian transposition respectively. $\|\mathcal{A}\|_{F}, \sqrt{\mathcal{A}}$ and $\operatorname{det}(\mathcal{A})$ denotes the Frobenius norm, Hermitian square root and determinant of matrix $\mathcal{A}$ respectively. Capital bold letters represent matrices, small underlined letters are vectors. Finally $\circledast$ denotes cyclic convolution and $Q(\cdot)$ denotes the $Q$-function.

\section{SYSTEM MODEL}

Figure 1 depicts a general structure of the new transmission scheme for OFDM system in a MIMO channel configuration employing $N_{t}$ and $N_{r}$ antennas at the transmitter and receiver, respectively. The OFDM system we consider in this paper utilizes $N_{c}$ frequency tones and the channel is assumed to be frequencyselective. At the transmitter, a sequence of $N_{s}$ baseband modulated data symbols $\{s(n)\}_{n=0}^{N_{s}-1}$ is first space-time (ST) encoded into codewords $\left\{\mathbf{C}_{k} \mid k=\right.$ $\left.1, \cdots, N_{c}\right\} \in \mathcal{C}^{p \times N_{t}}$, using $N_{t}$ antennas and $p$ adjacent OFDM symbols. Before the inverse Fourier transform (IFFT) operation, encoded subcarriers are split into a set of $N_{t}$ parallel symbol sequences and linearly transformed by the transmitter antenna weights before it is sent over the wireless channel. 


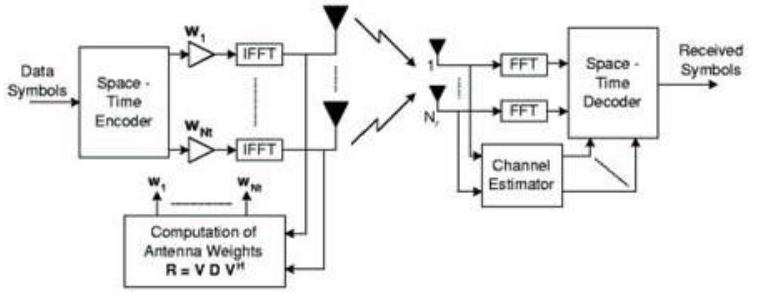

Fig. 1. Structure of OFDM system employing new adaptive transmit diversity scheme with transmit beamforming and ST encoding.

\subsection{Spatially Correlated Channel}

In this Subsection we assume that a uniform linear array (ULA) configuration is used for $N_{t}$ transmit antennas with a spacing of $d$ meters between adjacent antenna elements. In the reverse link (uplink), transmission occurs over a multipath channel environment. Let the $\ell^{\text {th }}$ path signal impinging on an ULA have an angle-of-arrival (AoA) of $\phi_{\ell}$. The array propagation vector can be found as $\underline{a}\left(\phi_{\ell}\right):=$ $\left[1, e^{j \beta}, e^{j 2 \beta}, \cdots, e^{j\left(N_{t}-1\right) \beta}\right]^{T}$ where $\beta=(2 \pi \cdot d$. $\left.\sin \left(\phi_{\ell}\right)\right) / \lambda$ and $\lambda$ being the carrier frequency wavelength. In general, the spacing between the elements of ULA at the transmitter is not large due to the condition at the antenna site to have zero cross-correlation factor, a typical scenario in cellular network. Therefore, spatial correlation between antenna elements would be effective. This correlation is defined in [4] based on the geometric scenario characterized by AoA. According to [4], the spatial covariance matrix that specifies the spatial correlation between antenna elements is given by:

$$
\mathbf{R}=\frac{1}{L} \sum_{\ell=1}^{L} \underline{a}\left(\phi_{\ell}\right) \underline{a}^{H}\left(\phi_{\ell}\right)
$$

where $L$ denotes the number of dominant resolvable paths. In this paper, we assume that spatial channel correlation at the receiver side is zero, hence, only the transmitter side will be considered. This means that $\mathbf{R}_{r}=I$, and hence we will use $\mathbf{R}$ to denote $\mathbf{R}_{t}$ (commonly used notation) throughout this paper.

\subsection{Antenna Weights Computation}

To maximize the transmitted signal power along the dominant multipaths, eigen-decomposition of the spatial covariance matrix should be performed, then we apply the resulting antenna weights given by the eigenvector that corresponds to the largest eigenvalue. The eigen-decomposition has the following form:

$$
\mathbf{R}=\mathbf{V D V}^{H}
$$

where $\mathbf{D}:=\operatorname{diag}\left(\mu_{d}, \mu_{2}, \cdots, \mu_{N_{t}}\right)$ is a diagonal matrix with ordered eigenvalues on the main diagonal and $\mathbf{V}$ is a unitary matrix composed of the corresponding eigenvectors. Hence, the transmit weight vector $\underline{w}:=\left[w_{1}, w_{2}, \cdots, w_{N_{t}}\right]^{T}$ can be found as the first column of $\mathbf{V}$ and the transmission on different eigenvectors will lead to uncorrelated channel fading.

\subsection{Received Signal Model}

Let the vector $\left\{\underline{x}_{i}^{n} \mid i=1, \cdots, N_{t}, n=0, \cdots, p-1\right\}$ contain the ST encoded symbols that are transmitted in parallel on $N_{c}$ frequency tones (carriers) by the $i^{\text {th }}$ transmit antenna during $n^{\text {th }}$ time interval, and let $w_{i}^{n}$ be the beamforming weight for the $i^{\text {th }}$ antenna. The discrete time model of the received signal at the $j^{\text {th }}$ receive antenna can be expressed as:

$$
\begin{aligned}
\underline{y}_{j}^{n}= & \sum_{i=1}^{N_{t}} \sum_{\ell=1}^{L} \operatorname{FFT}\left\{\operatorname{IFFT}\left\{\underline{x}_{i}^{n} w_{i}^{n}\right\} \circledast \underline{h}_{i, j}^{\ell, n}\right\} \\
& +\underline{\eta}_{j}^{n} \in \mathbb{C}^{N_{t} \times 1}
\end{aligned}
$$

Elements of the vector $\underline{\eta}_{j}^{n} \sim \mathcal{N}\left(0, \sigma_{n}^{2}\right)$ are modelled as uncorrelated additive white Gaussian random variables, and thus $\operatorname{FFT}\left(\underline{\eta}_{j}^{n}\right)$ is still white. The vector $\underline{h}_{i, j}^{\ell, n}$ represents the channel impulse response for the $l^{\text {th }}$ tap. In this paper, elements of this channel impulse response vector are modelled according to the geometrical-based hyperbolically distributed scatterers (GBHDS) channel model in [5] with Jakes power spectral density. It contains information about channel fading coefficient, multipath delay spread, Doppler spread, and angle spread.

If we transform the channel impulse response vector to the frequency domain, the frequency response of a spatially correlated channel in the $k^{\text {th }}$ tone at the time instant $n$ has the form:

$\mathbf{H}_{k, n}=\left[\underline{h}_{1, k, n}(1), \cdots, \underline{h}_{1, k, n}\left(N_{r}\right)\right] \in \mathbb{C}^{N_{t} \times N_{r}}$,

$\underline{h}_{1, k, n}(j)=\sum_{\ell=0}^{L-1} \sqrt{\mathbf{R}}\left[\begin{array}{c}h_{1, j}^{\ell, n}(k) e^{\frac{-j 2 \pi \tau_{\ell}(k-1)}{N_{c}}} \\ \vdots \\ h_{N_{t}, j}^{\ell, n}(k) e^{\frac{-j 2 \pi \tau_{\ell}(k-1)}{N_{c}}}\end{array}\right]$.

$\underline{h}_{i, j}^{\ell, n}(k)$ denotes the $k^{\text {th }}$ entry of the vector and $\tau_{\ell}$ is the delay of the $\ell^{\text {th }}$ path. Let us represent the antennas weights vector $\underline{w}$ as $\mathbf{W}_{n}:=\operatorname{diag}\left(w_{1}, w_{2}, \cdots, w_{N_{t}}\right)$, and apply $\mathbf{W}_{n}^{H}$ to the space-time encoded codeword $\mathbf{C}_{k}$ prior to transmission. Then the combined received signal at the receiver can be expressed as:

$$
\mathbf{Y}_{k}=\mathbf{C}_{k} \mathbf{W}^{H} \mathbf{H}_{k}+\mathbf{E}_{k}, k=1, \cdots, N_{c} .
$$

$\mathbf{E}_{k}$ is the receiver noise matrix. If we assume that channel fading coefficients remain constant for the duration of one block length $p$ (quasi-static fading), then the time index $n$ used in (5) can be taken out as in (6).

At the receiver, channel estimation is performed by evaluating pilot tones embedded in the transmitted signal, then it is fed into the maximum likelihood detector (MLD) for ST decoding of data symbols. We assume that the MS receiver has perfect channel knowledge. To decode the received signal matrix, the MLD will evaluate the decision matrix and decide in favor of the codeword as follows:

$$
\widehat{\mathbf{C}}=\arg \min _{\mathbf{C} \in \mathcal{C}} \sum_{k=1}^{N_{c}}\left\|\mathbf{Y}_{k}-\mathbf{C}_{k} \mathbf{W}^{H} \mathbf{H}_{k}\right\|_{F}^{2}
$$




\subsection{Pairwise Error Probability}

Based on the average SNR expression in [3] we can found that the SNR at the receive antennas has the form $\gamma=\sqrt{\mu_{d}} \varepsilon_{s} / \sigma_{n}^{2}$, where $\varepsilon_{s}$ denotes average symbol energy and $\sigma_{n}^{2}$ is the receiver noise variance. Let us assume that the receiver has perfect knowledge of the channel and the decision matrix in (7) is evaluated at the MLD for ST decoding. The pairwise error probability (PEP) for an erroneous detection of the codeword $\widetilde{\mathbf{C}}$ in favor of transmitted $\mathbf{C}$ is given by:

$$
P_{r}(\mathbf{C} \rightarrow \widetilde{\mathbf{C}} \mid \mathcal{H})=Q\left(\sqrt{\frac{d^{2}(\mathbf{C}, \widetilde{\mathbf{C}}) \gamma}{2}}\right)
$$

where $\mathcal{H} \triangleq\left\{\mathbf{W}^{H} \mathbf{H}_{1}, \cdots, \mathbf{W}^{H} \mathbf{H}_{N_{c}}\right\}$ and $d^{2}(\mathbf{C}, \widetilde{\mathbf{C}}):=\sum_{k=1}^{N_{c}}\left\|\mathbf{W}^{H} \mathbf{H}_{k}\left(\mathbf{C}_{k}-\widetilde{\mathbf{C}}_{k}\right)\right\|_{F}^{2}$ is commonly known as the squared Euclidean distance between the two codewords $\mathbf{C}$ and $\widetilde{\mathbf{C}}$. Using the Chernoff bound in [6], (8) can be upper bounded as:

$$
P_{r}(\mathbf{C} \rightarrow \widetilde{\mathbf{C}} \mid \mathcal{H}) \leq \exp \left(\frac{-d^{2}(\mathbf{C}, \widetilde{\mathbf{C}}) \gamma}{4}\right)
$$

\section{ACHIEVING TRANSMIT DIVERSITY}

For a system employing ST coding to achieve full diversity advantage, two major conditions must be satisfied [7]:

1. The energy of one information symbol must spread over at least as many time intervals as there are transmit antennas. Hence, the ST codeword matrix must have a block length $p \geq N_{t}$.

2. The propagation channel should be uncorrelated, i.e., channel fading coefficients are identical independently distributed (i.i.d.), this means that $\operatorname{rank}\left(\mathbf{H}_{k}\right)=N_{t}$.

However, correlation between signal paths always exists in practice; thus the second condition can never be met. Hence, to show that the proposed transmission structure decorrelates the channel covariance matrix (and hence maintain the maximum achievable diversity advantage of ST codes), the system must satisfy the following condition

$$
\operatorname{rank}\left\{\mathbf{W}^{H} \sqrt{\mathbf{R}}\right\}=N_{t} .
$$

In the following three Subsections we will consider two special cases as well as a typical case.

\subsection{Full Channel Correlation}

For the special case of fully correlated channel where $\mathbf{R}(i, j)=1 \forall i, j$, the proposed system cannot decorrelate signal paths since elements of $\mathbf{H}_{k}$ are all the same. The eigen-decomposition of $\mathbf{R}$ will lead to signal transmission with equal transmit power for all antennas, i.e., $\mathbf{W}=\operatorname{diag}\left(\sqrt{\rho / N_{t}}\right) \in \mathbb{R}^{N_{t} \times N_{t}}, \rho$ being the total transmit power constraint.

\subsection{No Channel Correlation}

Another spacial case: when there is no channel correlation we have $\mathbf{R}=I$. In such a case that satisfies Condition 2 above, no signal paths decorrelation can be performed, and thus the signal transmission should be done in the same way as in the special case of full channel correlation (with equal transmit power for all antennas): $\mathbf{W}=\operatorname{diag}\left(\sqrt{\rho / N_{t}}\right) \in \mathbb{R}^{N_{t} \times N_{t}}$. Therefore, no beamforming is utilized again in this case. However, full diversity order can still be obtained for the overall system with $\operatorname{rank}\left(\mathbf{W}^{H}\right)=\operatorname{rank}(\sqrt{\mathbf{R}})=N_{t}$.

\subsection{Partial Channel Correlation}

This is a typical scenario in the wireless channel environment. In general, without considering the first two idealistic situations, the channel covariance matrix $\mathbf{R}$ in (1) is a nonnegative definite Hermitian matrix of a Toeplitz form, i.e., $\mathbf{R}^{H}=\mathbf{R}$ and $\operatorname{det}(\mathbf{R})>0$. This means $\sqrt{\mathbf{R}}$ will always have full rank equals to $N_{t}$. Next, recall that the antenna weight matrix is the dominant eigenvector of the correlation matrix $\mathbf{R}$, then it also have a full rank equals to $N_{t}$. Thus, we can clearly see that the condition specified in (10) is satisfied, and hence this new transmission structure achieves full diversity order.

Therefore, except for the special case of full channel correlation, the above paragraph proved the claim that the proposed system can maintain the maximum achievable diversity order as long as the ST codeword matrix has the block length $p \geq N_{t}$.

\section{NUMERICAL RESULTS AND DISCUSSION}

In this section, we provide simulation results for the proposed transmission scheme in OFDM systems. In these simulations, the maximum Doppler frequency shift is $100 \mathrm{~Hz}$ and $N_{c}=512$ frequency tones are used for OFDM modulation. For ST coded OFDM, we use the coding scheme represented by that matrix $\mathcal{G} 4$ of OSTBC in [2] with QPSK modulated input symbols.

In these simulations, cyclic prefix (CP) is appended at the end of each OFDM word after the IFFT at the transmitter to avoid intersymbol interference (ISI). CP is discarded at the receiver before the FFT operation. We assume that the delay spread introduced by the frequency-selective channel is shorter than one CP length and thus converts wideband channel into frequency-flat fading for all $N_{c}$ frequency tones.

Figure 2 shows the performance of the OSTBCOFDM transmit diversity scheme in terms of SER and BER for different values of $N_{r}$ using ULA with and $N_{t}=4$ and element spacing of $0.5 \lambda$ at the transmitter. At an error rate of $10^{-2}$, the OSTBC-OFDM system have a $3.5 \mathrm{~dB}$ gain with an additional receiving antenna and a $5 \mathrm{~dB}$ gain at $10^{-4}$. If we compare these curves to the ones in Fig. 3 and Fig. 4 where OFDM systems are simulated in correlated channel environment, we observe that the error rate performance is 


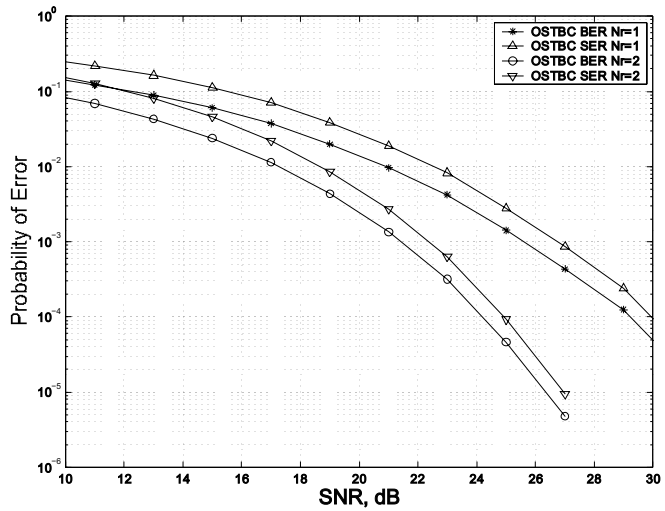

Fig. 2. Error performance curves of OSTBC OFDM system in uncorrelated fading channel with $N_{t}=4$.

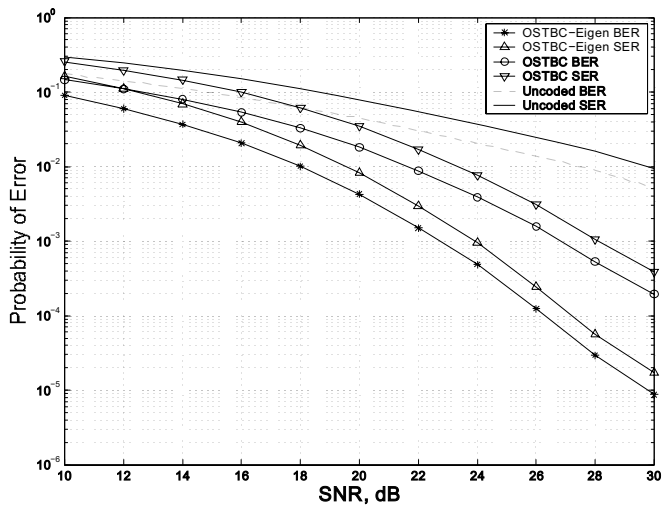

Fig. 3. Error performance curves of uncoded, OSTBC, and OSTBC-Eigen OFDM systems in GBHDS correlated fading channel with $N_{t}=4$ and $N_{r}=1$.

slightly lower when adaptive beamforming is not utilized. This shows that systems would perform worse in a spatially correlated channel due to coherent deep fades if no counter-measure techniques are used.

Figure 3 shows the error performance of OFDM systems employing different transmission schemes with $N_{r}=1$. Forward link channel with GBHDS is used for modelling a macrocell environment. At an error rate of $10^{-1}$, there is a $2.5 \mathrm{~dB}$ SNR improvement in OSTBC over the uncoded system, and an SNR gain of $3.5 \mathrm{~dB}$ in the proposed adaptive scheme (OSTBCEigen) over OSTBC. At the lower error rate of $10^{-3}$, a $4 \mathrm{~dB}$ gain is shown for the OSTBC-Eigen compared to OSTBC. The performance difference between OSTBC-Eigen and the other two schemes increases when SNR increases. Thus shows the decorrelation process gives better performance in an environment with a higher degree of spatial channel correlation.

Figure 4 shows the performance of OFDM systems employing different transmission schemes with $N_{r}=$ 2. In this case the error rate is lower than that in Fig. 3. Also, a significant gain in SNR of about $6.4 \mathrm{~dB}$ at an error rate of $10^{-5}$ for OSTBC-Eigen and $5 \mathrm{~dB}$ at an error rate of $10^{-3}$ for OSTBC are obtained in both SER and BER. This demonstrates that, when switching from a single receive antenna to two antennas, systems employing adaptive transmission scheme enjoy a diversity improvement of approximately $1.4 \mathrm{~dB}$ higher than that for systems using just OSTBC coding.

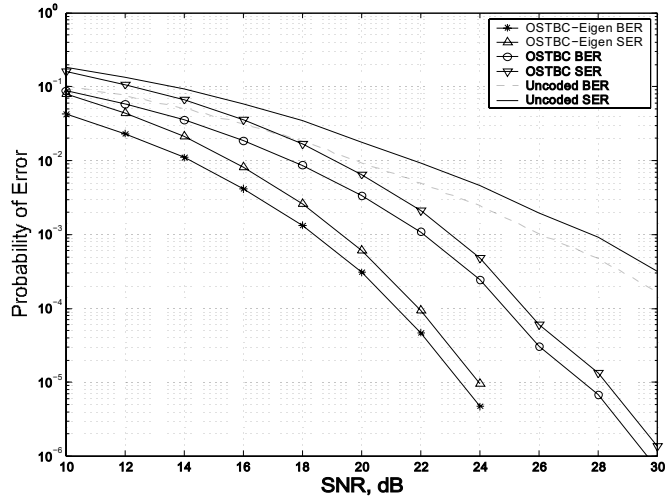

Fig. 4. Error performance curves of uncoded, OSTBC, and OSTBC-Eigen OFDM systems in GBHDS correlated fading channel with $N_{t}=4$ and $N_{r}=2$.

\section{CONCLUSIONS}

In this paper the performance of adaptive transmit diversity scheme that combines eigenbeamforming with OSTBC for OFDM systems has been investigated. With an effective utilization of spatial signal correlation at the multi-antenna transmitter, the performance of a predetermined ST code in OFDM systems can be improved. Simulation results demonstrated a significant performance improvement in terms of bit and symbol error rates over a spatially correlated channel model with different number of receiving antennas. Frequency-selective channels with Doppler effect are considered. It is also proved that systems utilizing this adaptive scheme will not be disadvantaged in terms of transmit diversity.

\section{REFERENCES}

[1] S. M. Alamouti, "A simple transmit diversity technique for wireless communications," IEEE J. Select. Areas Commun., vol. 16, pp. 1451-1458, Oct. 1998.

[2] V. Tarokh, H. Jafarkhani, and A. R. Calderbank, "Spacetime block codes from orthogonal designs," IEEE Trans. Inform. Theory, vol. 45, pp. 1456-1467, Jul. 1998.

[3] K. H. Lin, Z. M. Hussain, and R. Harris, "Adaptive transmit eigenbeamforming with orthogonal spacetime block coding in correlated space-time channels," in Proc. IEEE ICASSP, vol. 4, 2004, pp. 817-820.

[4] Siemens, Channel Model for Tx Diversity Simulations using Correlated Antennas, 3GPP Document TSG-RAN WG1 \#15, R1-00-1067, Berlin, Germany, Aug. 2000.

[5] S. S. Mahmoud, Z. M. Hussain, and P. O'Shea, "A space-time model for mobile radio channel with hyperbolically distributed scatterers," IEEE Antennas and Wireless Propagation Letters, vol. 1, pp. 211-214, 2002.

[6] J. G. Proakis, Digital Communications, New York, N.Y: McGraw-Hill Inc., Fourth Edition, 2001.

[7] E. G. Larsson and P. Stoica, Space-Time Block Coding for Wireless Communications, Cambridge, U.K.: Cambridge Univ. Press, 2003. 